University of Wollongong

Research Online

Australian Institute for Innovative Materials -

Papers

Australian Institute for Innovative Materials

$1-1-2014$

\title{
Platinum dendritic nanoparticles with magnetic behavior
}

Wenxian Li

University of Wollongong, wenxian@uow.edu.au

Ziqi Sun

University of Wollongong, ziqi@uow.edu.au

Dongliang Tian

University of Wollongong, dtian@uow.edu.au

Ivan P. Nevirkovets

University of Wollongong, ivann@uow.edu.au

S X. Dou

University of Wollongong, shi@uow.edu.au

Follow this and additional works at: https://ro.uow.edu.au/aiimpapers

Part of the Engineering Commons, and the Physical Sciences and Mathematics Commons

Research Online is the open access institutional repository for the University of Wollongong. For further information contact the UOW Library: research-pubs@uow.edu.au 


\title{
Platinum dendritic nanoparticles with magnetic behavior
}

\begin{abstract}
Magnetic nanoparticles have attracted increasing attention for biomedical applications in magnetic resonance imaging, high frequency magnetic field hyperthermia therapies, and magnetic-field-gradienttargeted drug delivery. In this study, three-dimensional (3D) platinum nanostructures with large surface area that features magnetic behavior have been demonstrated. The well-developed 3D nanodendrites consist of plentiful interconnected nano-arms $\sim 4 \mathrm{~nm}$ in size. The magnetic behavior of the 3D dendritic Pt nanoparticles is contributed by the localization of surface electrons due to strongly bonded oxygen/ Pluronic F127 and the local magnetic moment induced by oxygen vacancies on the neighboring Pt and $\mathrm{O}$ atoms. The magnetization of the nanoparticles exhibits a mixed paramagnetic and ferromagnetic state, originating from the core and surface, respectively. The 3D nanodendrite structure is suitable for surface modification and high amounts of drug loading if the transition temperature was enhanced to room temperature properly.
\end{abstract}

\section{Keywords}

dendritic, nanoparticles, magnetic, behavior, platinum

Disciplines

Engineering | Physical Sciences and Mathematics

\section{Publication Details}

Li, W., Sun, Z., Tian, D., Nevirkovets, I. P. \& Dou, S. (2014). Platinum dendritic nanoparticles with magnetic behavior. Journal of Applied Physics, 116 (3), 033911-1-033911-6. 


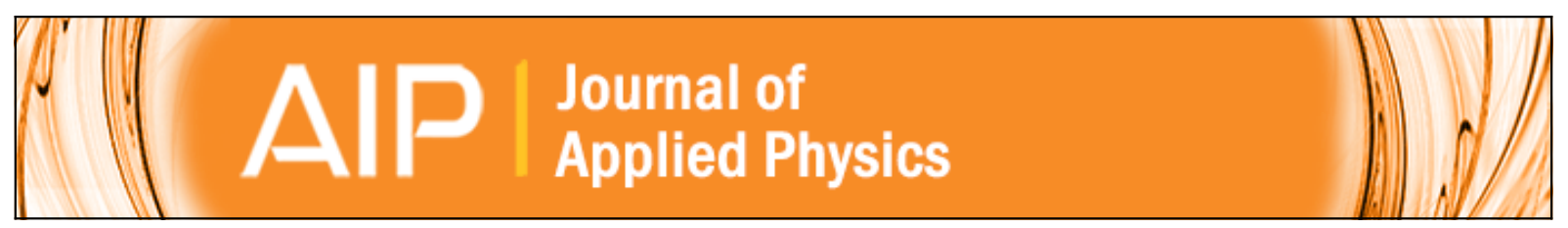

\section{Platinum dendritic nanoparticles with magnetic behavior}

Wenxian Li, Ziqi Sun, Dongliang Tian, Ivan P. Nevirkovets, and Shi-Xue Dou

Citation: Journal of Applied Physics 116, 033911 (2014); doi: 10.1063/1.4890506

View online: http://dx.doi.org/10.1063/1.4890506

View Table of Contents: http://scitation.aip.org/content/aip/journal/jap/116/3?ver=pdfcov

Published by the AIP Publishing

\section{Articles you may be interested in}

Room temperature ferromagnetism in defective $\mathrm{TiO} 2$ nanoparticles: Role of surface and grain boundary oxygen vacancies

J. Appl. Phys. 114, 203906 (2013); 10.1063/1.4833562

Room temperature ferromagnetism in non-magnetic doped $\mathrm{TiO} 2$ nanoparticles

J. Appl. Phys. 113, 17B511 (2013); 10.1063/1.4795615

Reversible switching of room temperature ferromagnetism in CeO2-Co nanoparticles

Appl. Phys. Lett. 100, 172405 (2012); 10.1063/1.4705045

Magnetic properties of $\mathrm{ZnFe} 2 \mathrm{O} 4$ ferrite nanoparticles embedded in $\mathrm{ZnO}$ matrix Appl. Phys. Lett. 100, 122403 (2012); 10.1063/1.3696024

Enhanced indirect ferromagnetic p-d exchange coupling of $\mathrm{Mn}$ in oxygen rich ZnO:Mn nanoparticles synthesized by wet chemical method

J. Appl. Phys. 111, 033503 (2012); 10.1063/1.3679129

\section{AlP $\left.\right|_{\text {Applied Physics }} ^{\text {Journal of }}$}

Journal of Applied Physics is pleased to announce André Anders as its new Editor-in-Chief 


\title{
Platinum dendritic nanoparticles with magnetic behavior
}

\author{
Wenxian Li, ${ }^{1,2, a)}$ Ziqi Sun, ${ }^{1}$ Dongliang Tian, ${ }^{3}$ Ivan P. Nevirkovets, ${ }^{1}$ and Shi-Xue Dou ${ }^{1}$ \\ ${ }_{1}^{1}$ Institute for Superconducting and Electronic Materials, University of Wollongong, NSW 2522, Australia \\ ${ }^{2}$ Solar Energy Technologies, School of Computing, Engineering, and Mathematics, University of Western \\ Sydney, Penrith NSW 2751, Australia \\ ${ }^{3}$ Key Laboratory of Bio-Inspired Smart Interfacial Science and Technology of the Ministry of Education, \\ School of Chemistry and the Environment, Beihang University, Beijing 100191, People's Republic of China
}

(Received 16 February 2014; accepted 6 July 2014; published online 17 July 2014)

\begin{abstract}
Magnetic nanoparticles have attracted increasing attention for biomedical applications in magnetic resonance imaging, high frequency magnetic field hyperthermia therapies, and magnetic-fieldgradient-targeted drug delivery. In this study, three-dimensional (3D) platinum nanostructures with large surface area that features magnetic behavior have been demonstrated. The well-developed 3D nanodendrites consist of plentiful interconnected nano-arms $\sim 4 \mathrm{~nm}$ in size. The magnetic behavior of the 3D dendritic Pt nanoparticles is contributed by the localization of surface electrons due to strongly bonded oxygen/Pluronic F127 and the local magnetic moment induced by oxygen vacancies on the neighboring $\mathrm{Pt}$ and $\mathrm{O}$ atoms. The magnetization of the nanoparticles exhibits a mixed paramagnetic and ferromagnetic state, originating from the core and surface, respectively. The 3D nanodendrite structure is suitable for surface modification and high amounts of drug loading if the transition temperature was enhanced to room temperature properly. (C 2014 AIP Publishing LLC.

[http://dx.doi.org/10.1063/1.4890506]
\end{abstract}

\section{INTRODUCTION}

The huge potential applications of magnetic nanoparticles have attracted much interest among magnetism researchers who are working to advance the quality and reliability in recording technology, biology, and medicine. ${ }^{1,2}$ Controlled drug release from drug-loaded nanoparticle capsules, especially loaded on magnetic nanoparticles, is attracting increasing attention because the high efficiency of drug delivery in cancer therapy can make it possible to avoid to hurting healthy cells. The magnetic nanoparticles can be employed in several practical medical and biological applications. They can be used as magnetic contrast agents in magnetic resonance imaging (MRI), hyperthermia agents to ablate the diseased cells under high frequency magnetic field, and magnetic vectors to realize targeted drug delivery that is directed by means of a magnetic field gradient towards a certain location. ${ }^{3}$ Nanotechnology has generated many salient electronic, optical, and magnetic properties in noble metal nanoparticles through morphology modification on the nanoscale, such as by changing the size, shape, and geometry. ${ }^{4,5}$ Besides the application of noble nanomaterials in catalysis, sensors, electrochemistry, photoelectric conversion, and surface enhanced Raman spectroscopy, the magnetism in noble metal avoids the great disadvantage of normal metallic nanoparticles of being pyrophoric and reactive to oxidizing agents to various degrees. They have been predicted to revolutionize medicine, with uses in biomolecule recognition, drug release control, and cancer treatment. ${ }^{6,7}$ Noble metal nanoparticles are widely employed in a variety of biomedical applications, such as in highly sensitive

\footnotetext{
a) Author to whom correspondence should be addressed. Electronic mail: w1240@uowmail.edu.au
}

diagnostic assays, ${ }^{8}$ radiotherapy enhancement, ${ }^{9}$ and drug and gene delivery. ${ }^{10,11}$ Nanotechnology has allowed us to develop efficient methods to diagnose and treat cancer, such as with novel magnetic imaging agents, multifunctional, targeted devices for delivering therapeutic agents to cells and tissues infiltrated by cancer, and ways of monitoring predictive molecular changes and minimizing costs and side effects. ${ }^{12}$ Furthermore, noble metal nanoparticles are nontoxic agents for drug and gene delivery applications. ${ }^{13}$ Ultrathin nanowires or nanoclusters with dimensions of a few atoms show a reduced degree of coordination, and their bonding favors more localized electronic states. These features will induce narrower bands and larger densities of states at the Fermi level to generate exotic magnetic behavior. ${ }^{14}$ The bulk $4 \mathrm{~d}$ and $5 \mathrm{~d}$ noble metals, such as palladium $(\mathrm{Pd})$, platinum $(\mathrm{Pt})$, and gold $(\mathrm{Au})$, are diamagnetic or paramagnetic, although two-dimensional (2D) atomic layers ${ }^{15-17}$ or zero-dimensional (0D) nanoclusters of these metals ${ }^{6,18-22}$ have been shown experimentally to be magnetic. Saturation magnetization values of up to $13 \mathrm{~A} \mathrm{~m}^{2} \mathrm{~kg}^{-1}$ have been reported, which is about $5 \%$ of that of bulk iron. ${ }^{23}$ Most of the reported nanoparticles display as-dispersed polyhedra or one-dimensional (1D) nanowires or nanorods, which are not suitable for loading with medicines due to their smooth surfaces. Magnetic noble metal nanostructures with large surface area, however, will be practical carriers for drug delivery with high loading capacity.

Herein, we propose a simple one-step synthesis for uniform-sized three-dimensional (3D) Pt nanodendrites with a very fine particle size from an aqueous surfactant solution at room temperature, without the need for any template, seed-mediated growth, or additive. ${ }^{24}$ The size of the obtained Pt nanodendrites ranges very narrowly from 50 to $70 \mathrm{~nm}$, with $60 \mathrm{~nm}$ being the predominant diameter. The individual 
Pt particles that make up the nanodendrites present a uniform size of around $4 \mathrm{~nm}$ in diameter. The nanodendrites show obvious room temperature magnetism. The oxidation state of the surface Pt and the electron behavior are discussed to clarify the origins of the anomalous magnetism in the $\mathrm{Pt}$ nanodendrites.

\section{EXPERIMENTAL DETAILS}

The synthesis of Pt nanodendrites was modified from Wang's report. ${ }^{25,26}$ In a typical synthesis process, $5 \mathrm{ml}$ of $20 \mathrm{mM} \quad \mathrm{H}_{2} \mathrm{PtCl}_{6}$ aqueous solution containing $0.8 \mathrm{mM}$ Pluronic F127 (polyethylene oxide (PEO)—polypropylene oxide (PPO) - polyethylene oxide block copolymer with an average molecular structure of $\mathrm{PEO}_{100} \mathrm{PPO}_{65} \mathrm{PEO}_{100}$ and a molecular weight of 12600) was placed in a small bottle, and then $5 \mathrm{ml} 0.1 \mathrm{M}$ ascorbic acid was quickly added. The mixed solution was then put into an ultrasonic cleaner with a water temperature of $45^{\circ} \mathrm{C}$ for $45 \mathrm{~min}$. The color of the reaction solution gradually changed within $30 \mathrm{~min}$ from transparent light brownish-yellow to brown and then to opaque black during the Pt deposition process. The residual Pluronic F127 was removed by washing with water after the Pt deposition. Then, the powder was dried at $120^{\circ} \mathrm{C}$ in ambient air to remove the adsorbed $\mathrm{H}_{2} \mathrm{O}$ molecules.

The microstructure of the sample was characterized and analysed by X-ray diffraction (XRD: GBC MMA, Cu K , $\lambda=0.154056 \mathrm{~nm}$ ), X-ray photoelectron spectroscopy (XPS: PHOIBOS 100 Analyser from SPECS; Al $\mathrm{K}_{\alpha} \mathrm{X}$-rays), and transmission electron microscopy (TEM: JEOL-2010) with high resolution TEM (HRTEM) using $200 \mathrm{kV}$. Selected area electron diffraction (SAED) patterns were also collected for crystal structure analysis. Magnetic properties were measured using a commercial vibrating sample magnetometer (VSM) in a magnetic properties measurement system (MPMS: Quantum Design, 14 T), in applied magnetic fields up to $70 \mathrm{kOe}(7 \mathrm{~T})$ at $5 \mathrm{~K}$ and $40 \mathrm{kOe}(4 \mathrm{~T})$ at $305 \mathrm{~K}$. The nanoparticles were solidified with resin and packed into a polypropylene powder holder, which is an injection molded plastic part designed for use as a powder container during the VSM measurement process. The polypropylene powder holder was mounted in a brass trough, which was made from cartridge brass tubing with a cobalt-hardened gold plated finish. Both the polypropylene powder holder and the brass trough were made by Quantum Design as commercial VSM sample holders with very low diamagnetic moments, which are much lower than the moments of the Pt samples.

\section{RESULTS AND DISCUSSION}

Figure 1 shows the indexed XRD pattern of the Pt nanodendrites, in which no impurities can be detected. The XRD analysis indicates a face-centered cubic (FCC) structure having $F m \overline{3} m$ (group 225 ) symmetry. The diffraction peak positions and the corresponding lattice parameters are consistent with the standard values obtained from the Joint Committee on Powder Diffraction Standards (JCPDS: 70-2057), confirming that the samples are pure noble metal. The crystallite size for the platinum nanocrystals as determined by the Scherrer formula is $4.2 \mathrm{~nm}$.

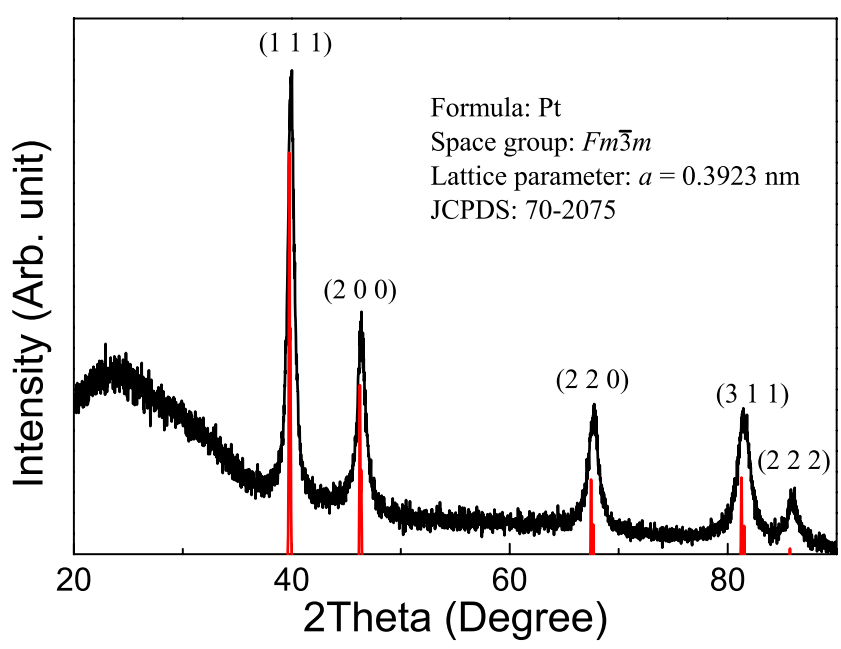

FIG. 1. Indexed XRD pattern of Pt nanodendrites shows broadened peaks due to the fine particle size.

The morphology of the typically synthesized product was characterized by TEM, as shown in Figure 2. The image obtained at lower magnification in Figure 2(a) indicates that the as-synthesized product features well-dispersed nanoparticles with complete dendritic shapes. The size of the nanoclusters ranges narrowly from 50 to $70 \mathrm{~nm}$ with $60 \mathrm{~nm}$ the predominant diameter. The surface area can reach $47 \mathrm{~m}^{2} \mathrm{~g}^{-1}$ in a similar structure from analysis of the $\mathrm{N}_{2}$ adsorption-desorption isotherm. ${ }^{25}$ The selected area electronic diffraction (SAED) pattern shown in the inset of Figure 2(a) features several diffraction rings assignable to FCC Pt crystals, which are consistent with the XRD pattern of the sample. The higher-magnification images shown in Figures 2(b) and 2(c) reveal that the well-developed nanodendrites consist of plentiful interconnected nano-arms around $4 \mathrm{~nm}$ in width. A highly magnified TEM image of one nanodendrite

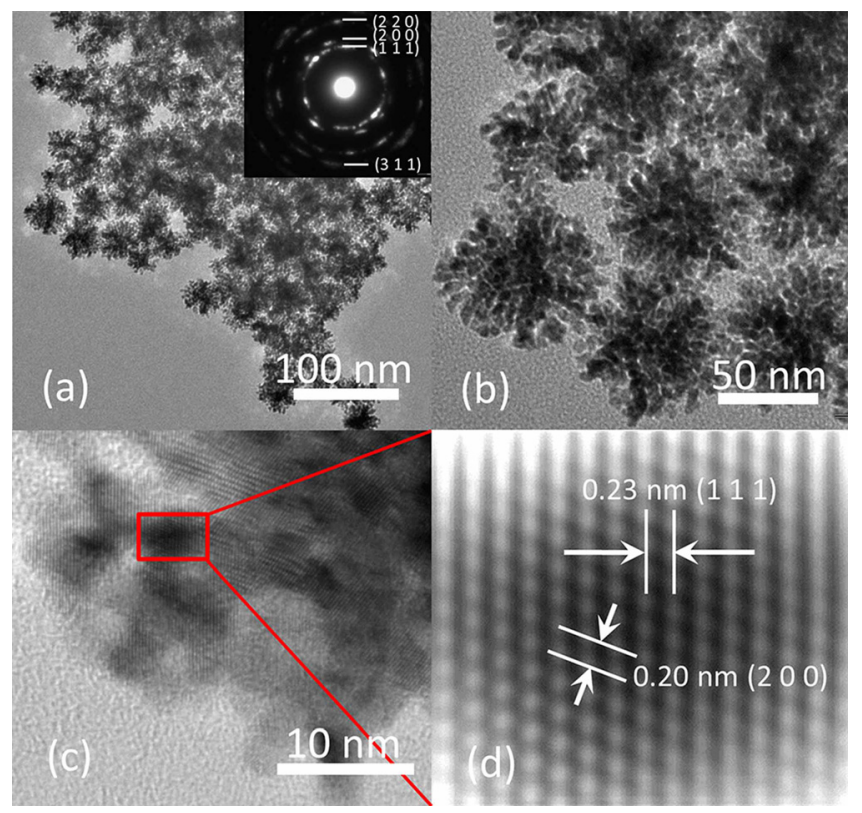

FIG. 2. (a)-(c) TEM images of Pt nanodendrites with at different magnifications. The inset of (a) shows the indexed polycrystalline SAED pattern. (d) High-resolution TEM image of the individual Pt nanoparticles shown in (c). 


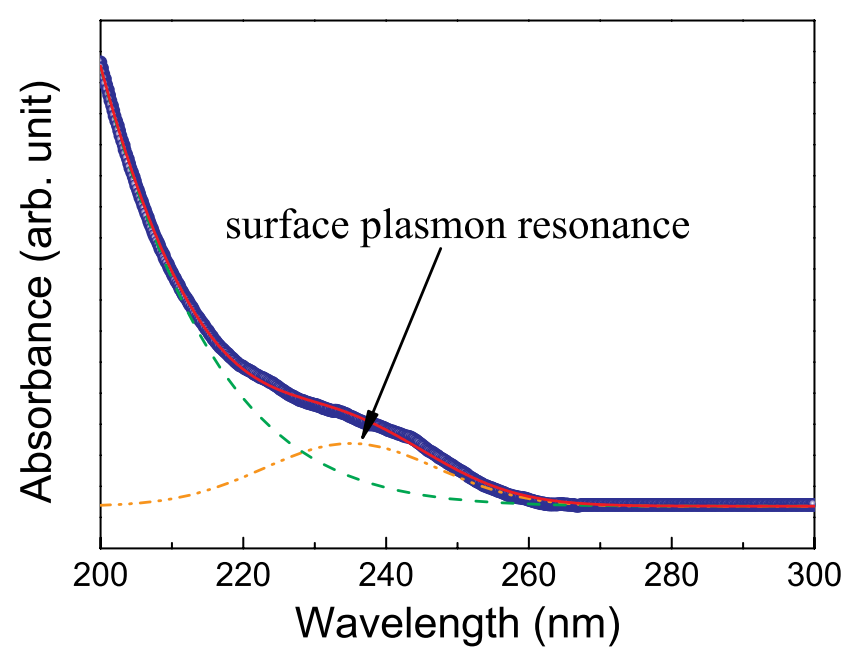

FIG. 3. Optical absorption spectrum of Pt nanodendrites displaying the surface plasmon resonance band at $\sim 235 \mathrm{~nm}$ with a width of $\sim 24 \mathrm{~nm}$.

[Figure 2(c)] further visualizes the interconnected nano-arms branching in various directions. The high resolution TEM image indicates that the lattice fringes are coherently extended across several nano-arms, as shown in Figure 2(d). The observed $d$-spacing $(0.23 \mathrm{~nm})$ between the adjacent fringes corresponds to the $\{111\}$ planes of the Pt FCC structure.

Figure 3 shows the optical absorption spectra of Pt nanodendrites, where a band at $235 \mathrm{~nm}$ can be observed as a shoulder over the whole range of the nanodendrite. This band is ascribed to the surface plasmon resonance effect, which originates from a collective oscillation of the conduction electrons inside the nanoparticle. ${ }^{19}$ The width of an absorption band is strongly dependent on the oscillation range of the electrons in the metal particles. The Pt nanodendrites have an absorption band of $\sim 24 \mathrm{~nm}$, which is six times longer than for the primary Pt particles, $\sim 4 \mathrm{~nm}$, according to the TEM images. The long electron oscillation range comes from the well-connected nanostructure of the $\mathrm{Pt}$ dendrites and the high mobility of the delocalized electrons.

The surface sensitive XPS technique was employed to examine the trace components, which cannot be detected by $\mathrm{XRD}$, and the surface states of the $\mathrm{Pt}$ nanodendrites, as shown in Figure 4. The survey scan displays the detectable elements Pt, Ar, O, and C, as shown in Figure 4(a). The element content is estimated basing on the peak area $(A)$ and relative sensitivity factor $(F): C_{m}=A_{m} F_{m} / \Sigma A_{i} F_{i}$, where the $F$ values of $\mathrm{O} 1 \mathrm{~s}, \mathrm{C} 1 \mathrm{~s}, \mathrm{Pt} 4 \mathrm{~d}$, and $\mathrm{Ar} 2 \mathrm{p}$ are 3.1, 1, 19.38, and 3.04, respectively. Ar is used to wash the vacuum chamber. Adventitious carbon is used as a reference during XPS measurements to calibrate the binding energy scale. The main component of the $\mathrm{C} 1 \mathrm{~s}$ peak is assumed to be positioned at $284.5 \mathrm{eV}$ for this purpose, as shown in Figure 4(b). The weak peak in Figure 4(b) may be attributed to remnant Pluronic F127, which is about 5.70 at. $\%$ in the total materials. The $\mathrm{O}$ 1s peak overlaps with the $\mathrm{Pt} 4 \mathrm{p} 3 / 2$ peak, as indicated by the dashed circle in Figure 4(a). The O 1s peak [see Figure 4(c)] is positioned at the binding energy of oxygen, $532.1 \mathrm{eV}$. The oxygen content is 20.75 at. \% in the sample, which can be attributed to both Pluronic F127 ( 6.39 at. \%, based on the ratio of carbon and oxygen, 7:3) and $\mathrm{PtO}_{x}[\sim 14.36$ at. \%, see the fitting results for $\mathrm{Pt} 4 \mathrm{f}$ in Figure 4(d)]. Actually, the content of Pluronic F127 molecules is not high in the Pt nanodendrites because every Pluronic F127 molecule has about 600 carbon atoms.
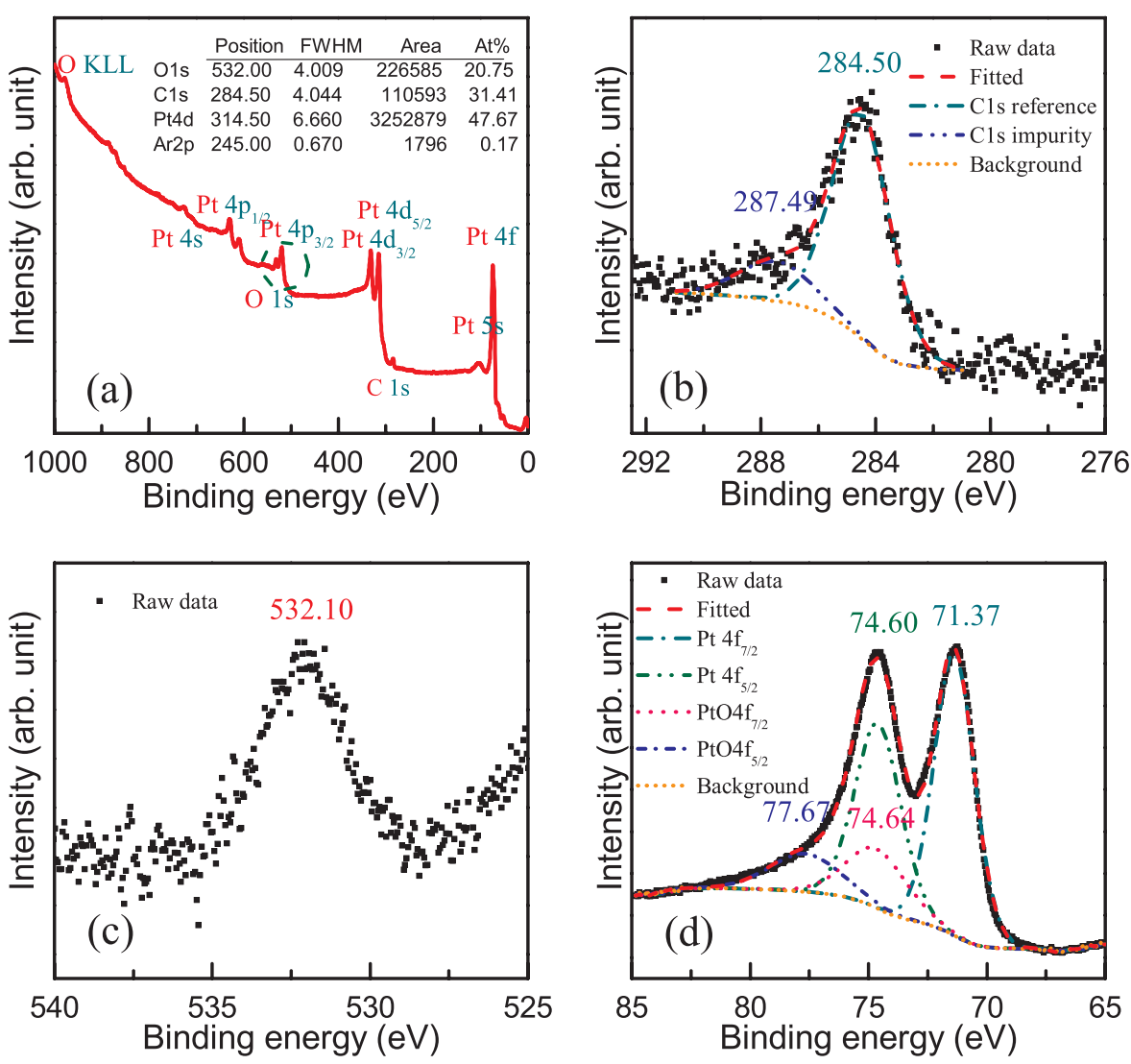

FIG. 4. (a) XPS survey scan of Pt nanodendrites and fitted results of (b) $\mathrm{C} 1 \mathrm{~s}$, (c) $\mathrm{O} 1 \mathrm{~s}$, and (d) Pt $4 \mathrm{f}$ peaks. From the ionization states of the Pt atoms on the surface, it was concluded that the Pt was present in the form of $\mathrm{PtO}_{x}$, a mixture of $\mathrm{PtO}$ and $\mathrm{Pt}_{2} \mathrm{O}_{3}$ in the ratio of $2: 1$. 

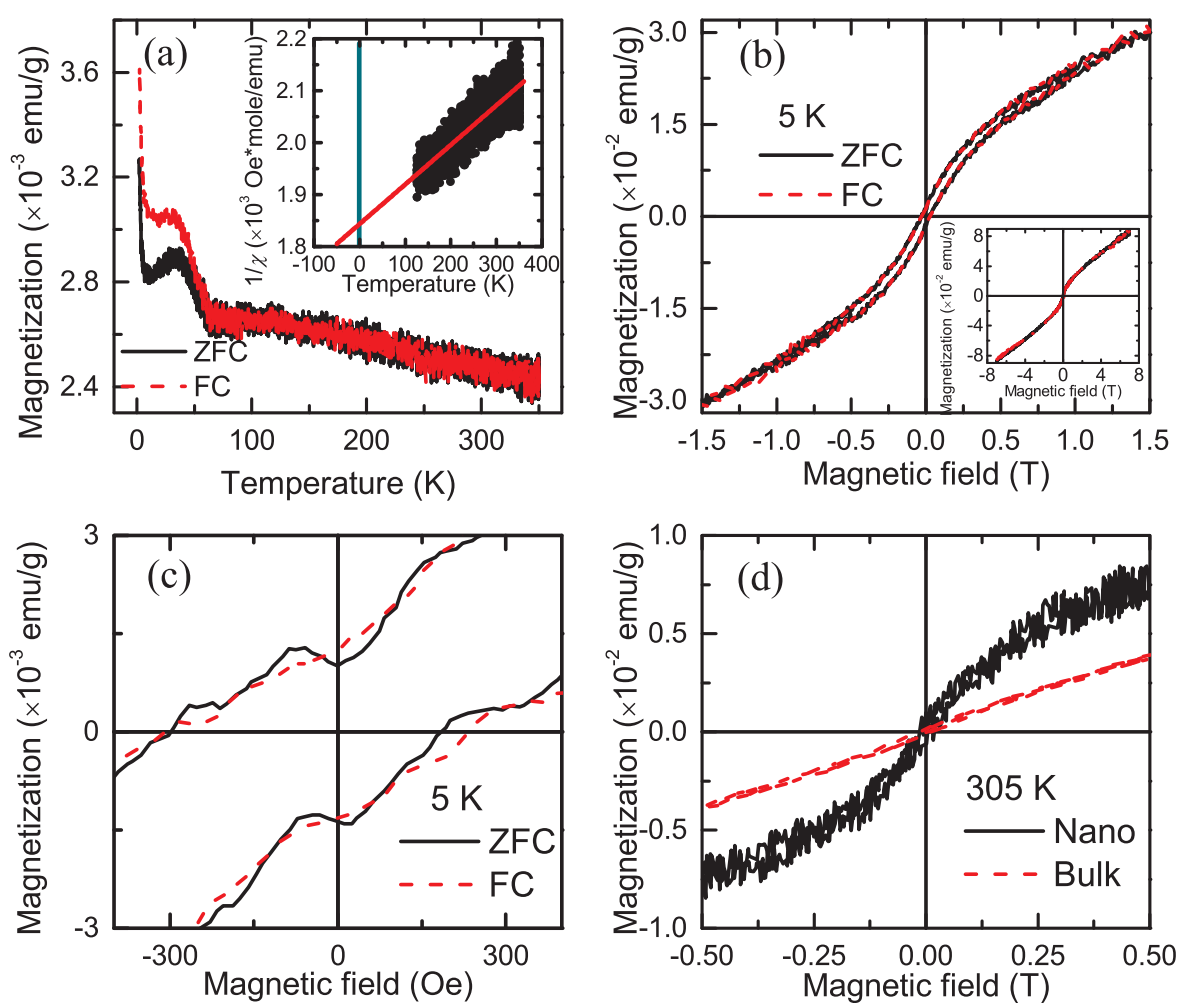

FIG. 5. (a) Magnetization dependence on temperature of $\mathrm{Pt}$ nanodendrites. $M(T)$ vs. $T$ curves after $\mathrm{ZFC}$ and after FC indicate the mixed paramagnetic and antiferromagnetic state. Both measurements were from $5 \mathrm{~K}$ to $350 \mathrm{~K}$ under $1 \mathrm{kOe}$ magnetic field. The CurieWeiss fitting result in the inset indicates the antiferromagnetic contribution. (b) Hysteresis loops measured at $5 \mathrm{~K}$ after $\mathrm{ZFC}$ and after FC under 1 kOe magnetic field from 350 to $5 \mathrm{~K}$ between $\pm 1 \mathrm{~T}$. The inset displays the whole hysteresis loops between $\pm 7 \mathrm{~T}$. (c) Residual magnetization and coercive field after ZFC and FC at $5 \mathrm{~K}$. (d) Hysteresis loop measured at $305 \mathrm{~K}$, indicating the magnetic field response of the Pt nanodendrites compared with the paramagnetic nature of bulk Pt. The hysteresis loop deviates from the linear behavior of paramagnetic bulk Pt.
XPS was also employed to obtain information on the oxidation states of the platinum nanodendrites and their relative contents. The fitted $\mathrm{Pt} 4 \mathrm{f}$ electron spectrum is shown in Figure 4(d). It was assumed that the doublets $(5 / 2-7 / 2)$ have Gaussian-Lorentzian (7:3) shaped components with an intensity ratio of $3: 4$ and a separation of about $3.30 \mathrm{eV}$. The Pt $4 \mathrm{f}$ spectrum was well-resolved with two $\mathrm{Pt} 4 \mathrm{f}_{7 / 2}$ binding energies centered at 71.37 and $74.64 \mathrm{eV}$ and two $\mathrm{Pt} 4 \mathrm{f}_{5 / 2}$ binding energies centered at 74.6 and $77.67 \mathrm{eV}$. The doublet at lower binding energies is undoubtedly due to free $\operatorname{Pt}(0),{ }^{24}$ while the higher binding energy component is due to oxidized $\mathrm{PtO}_{x}$. The $\mathrm{PtO}_{x}$ content is about 23.85 at. \% in the total $\mathrm{Pt}$ and about 11.37 at. \% in the XPS sample. It can be found that the content ratio of $\mathrm{Pt}$ to $\mathrm{O}$ is about 4:5. Thus, we can assume that $\mathrm{PtO}_{x}$ is a mixture of $\mathrm{PtO}$ and $\mathrm{Pt}_{2} \mathrm{O}_{3}$ in the ratio of 2:1. Nevertheless, the binding energy of $\mathrm{Pt} 4 \mathrm{f}_{7 / 2}$, $74.64 \mathrm{eV}$, is much higher than the reported result for $\mathrm{PtO}$, $72.2 \mathrm{eV},{ }^{24,27}$ and $\mathrm{Pt}_{2} \mathrm{O}_{3}, 73.6 \mathrm{eV},{ }^{24}$ but quite similar to that of $\mathrm{PtO}_{2}, 74.2 \mathrm{eV} .^{24,28}$ The positive shift in binding energy corresponds to a decrease in the electronic charge density on the surface of the $\mathrm{Pt}$ nanodendrites. This might arise from Pluronic $\mathrm{F} 127 / \mathrm{PtO}_{2-\delta}-\mathrm{Pt}$ or core-shell interactions, where there might be an electron shift from the surface to the Pluronic F127, as on the surface of thiol-capped Au nanoparticles, ${ }^{29}$ or to the core, as predicted in Ag clusters. ${ }^{30}$ Then, the $\mathrm{PtO}_{x}$ can be assumed to be $\mathrm{PtO}_{2-\delta}$. This phenomenon means that the surface of Pt nanodendrites is in a high oxidation state with a great oxygen shortage due to the fine size effect.

The magnetization dependence on temperature $M(T)$ is shown in Figure 5(a). Both zero-field-cooling (ZFC) effects and field-cooling (FC) effects were measured under 1000 Oe magnetic field, which is higher than the coercive field, as shown in Figures 5(b)-5(d), to avoid nanoparticle alignment during the measurement. The noisy signals induced by the alignment could not be eliminated in low magnetic field, even when the nanoparticles were fixed with resin, due to their ultra-fine size. The ZFC and FC curves did not show any obvious difference above $50 \mathrm{~K}$. The high temperature susceptibility $\left(\chi(T)=M(T) / \mu_{0} H\right.$, with $M(T)$ the magnetization dependence on temperature and $H$ the applied magnetic field) for the Pt bulk sample should follow the Curie-Weiss law: $1 / \chi(T)=(T-\theta) / C$, where $\theta$ is the Curie-Weiss temperature and $C$ is the Curie-Weiss constant. The measured $M(T)$ deviates greatly from the Curie-Weiss law, however, due to the confined size effects, and the transition becomes very complex. The magnetization increased gradually with decreasing temperature from $350 \mathrm{~K}$ to $\sim 70 \mathrm{~K}$. Then, the magnetization was significantly enhanced below $70 \mathrm{~K}$ due to the antiferromagnetic transition of $\mathrm{PtO}_{2}$. Yang et al. predicted the magnetism of $\mathrm{PtO}_{2}$ based on density functional theory (DFT) using the generalized gradient approximation with a Hubbard U method. ${ }^{31}$ They found that oxygen vacancy will induce local magnetic moments on the neighboring $\mathrm{Pt}$ and $\mathrm{O}$ atoms. The magnetism originates not only from the unpaired electrons that occupy the vacancy-induced gap states, but also from the itinerant valence electrons. This transition was not observed in previous reports. ${ }^{6,7,32}$

Figure 5(b) shows the hysteresis loops measured at $5 \mathrm{~K}$ after ZFC and FC under $1 \mathrm{kOe}$ magnetic field from 350 to $5 \mathrm{~K}$, before the hysteresis loops were measured between $\pm 7 \mathrm{~T}$, as shown in the inset. The magnetization was not saturated even at $\pm 7 \mathrm{~T}$ because of the paramagnetism of $\mathrm{Pt}$. In contrast to Pt bulk samples, obvious magnetic behavior was detected from the magnetic measurement. The hysteresis behavior was very clear between $\pm 1 \mathrm{~T}$, as shown in Figure 5(b). Figure 5(c) displays the coercive field and remanence. It should be noted there was no obvious difference between 
the hysteresis loops after ZFC and FC. Another interesting phenomenon was the room temperature magnetism in the $\mathrm{Pt}$ nanodendrites, as shown in Figure 5(d). For comparison, the paramagnetic loop of bulk $\mathrm{Pt}$ is also displayed in Figure 5 (d). The hysteresis loop was measured between $\pm 4 \mathrm{~T}$, and Figure 5(d) shows the low magnetic field part. It should be noted that the commercial copper sample holder and polypropylene powder holder were used in the measurements, and their diamagnetic moments are very weak compared with the paramagnetic signals of bulk Pt or the strong magnetic moments of Pt nanodendrites. The signals of the Pt nanodendrites were quite noisy and did not show any obvious coercive field or remanence, while the loop deviated from the linear behavior characteristic of paramagnetism. The loop above $\sim 2500$ Oe is parallel to that of the bulk sample. A comparison of the magnetic moments at $305 \mathrm{~K}$ on the $\mathrm{ZFC} / \mathrm{FC}$ curves with the magnetic moments on the hysteresis loop when the applied field was $1 \mathrm{kOe}$ indicates that the deviation comes from the nanoparticle alignment in the increasing magnetic field rather than from stable magnetic moments, which is consistent with the $\mathrm{ZFC} / \mathrm{FC}$ behavior above $50 \mathrm{~K}$.

The magnetic behavior is mostly attributed to surface effects or size effects because the crystal structure of the nanodendrites in this work is the same as for the Pt bulk, as indicated by the XRD pattern. The surface of the Pt nanodendrites is in a high oxidation state with great oxygen shortage, as indicated by the XPS spectrum. The stable magnetism in Pt nanodendrites possibly comes from the two sources below: (a) the localization of surface electrons by strongly bonded oxygen/Pluronic F127 and (b) the local magnetic moments induced by the oxygen vacancies among the neighboring $\mathrm{Pt}$ and $\mathrm{O}$ atoms.

As for the first reason, the active surface electrons are trapped by the strongly electronegative oxygen atoms. Although the bond is very strong, the oxygen atoms are only attached on the surface because there was no crystal transition. Otherwise, the $\mathrm{PtO}_{2-\delta}$ structure would be detectable from the XRD pattern due to its high content, 23.85 at. \% in the total Pt. These results are quite similar to what is seen with surface-decorated noble metal nanoparticles, ${ }^{29,32}$ in which the surface electrons show localized behavior. The optical absorption spectrum indicates that there are also delocalized electrons in the system and only part of the surface electrons contribute to the magnetic moments. The delocalized electrons can mediate the coupling of the unpaired electrons, however.

As to the second reason, the electronic structure of small metal clusters is sensitive to defects, especially the oxygen vacancy. Although the atomic ratio of $\mathrm{Pt}$ to $\mathrm{O}$ is $2: 3$ on the surface, the XPS spectrum of Pt $4 \mathrm{f}$ indicates that the Pt has lost more electrons than the demands of oxygen saturation absorption. Although the Pt nanodendrites did not show any special topological crystal structure, judging from the XRD pattern, the high oxidation state of the surface Pt confirms the fact of oxygen vacancy. According to first-principles calculations based on DFT, ${ }^{31}$ the oxygen vacancy induces a spin-polarized ground state. The three nearest-neighbor Pt atoms of the vacancy site show strong local magnetic moments, which originate from the occupation and reorganization of $5 d$ electrons due to the Coulomb interaction involving electrons transferred from the vacancy site. The net magnetic moment comes from antiferromagnetic coupling between the unpaired electrons in the vacancy state and the itinerant valence electrons. ${ }^{31}$

\section{CONCLUSIONS}

In summary, highly dispersed Pt nanodendrites with magnetic performance and large surface area were synthesized using a one-step reduction process. The surface of the Pt nanodendrites was in a high oxidation state, with a great oxygen shortage due to the fine size effect. The magnetization dependence on temperature deviates from the CurieWeiss law. The magnetic behavior of the Pt nanodendrites can be mainly attributed to two different phenomena involving the electrons: the localization of surface electrons by strongly bonded oxygen/Pluronic F127 and the oxygenvacancy-induced local magnetic moment on the neighboring $\mathrm{Pt}$ and $\mathrm{O}$ atoms. The magnetic moment may be enhanced by other types of contact with polymers with a strong electron transfer effect.

\section{ACKNOWLEDGMENTS}

The authors acknowledge support from Australian Research Council (ARC DP0879070). W.X.L. also acknowledges the financial support of a Vice Chancellor's Research Award from the University of Wollongong. We are grateful to Dr. Tania Silver for many fruitful conversations and her expert advice.

${ }^{1}$ B. D. Terris and T. Thomson, J. Phys. D: Appl. Phys. 38, R199 (2005).

${ }^{2}$ A. K. Gupta and M. Gupta, Biomaterials 26, 3995 (2005).

${ }^{3}$ M. Arruebo, R. Fernández-Pacheco, M. R. Ibarra, and J. Santamaría, Nano Today 2, 22 (2007).

${ }^{4}$ L. He, M. D. Musick, S. R. Nicewarner, F. G. Salinas, S. J. Benkovic, M. J. Natan, and C. D. Keating, J. Am. Chem. Soc. 122, 9071 (2000).

${ }^{5}$ A. N. Shipway, M. Lahav, and I. Willner, Adv. Mater. 12, 993 (2000).

${ }^{6}$ M. A. Garcia, M. L. Ruiz-Gonzalez, G. F. de la Fuente, P. Crespo, J. M. Gonzalez, J. Llopis, J. M. Gonzalez-Calbet, M. Vallet-Regi, and A. Hernando, Chem. Mater. 19, 889 (2007).

${ }^{7}$ X. W. Teng, M. Feygenson, Q. Wang, J. Q. He, W. X. Du, A. I. Frenkel, W. Q. Han, and M. Aronson, Nano Lett. 9, 3177 (2009).

${ }^{8}$ S. T. Selvan, T. T. Y. Tan, D. K. Yi, and N. R. Jana, Langmuir 26, 11631 (2010).

${ }^{9}$ X. H. Huang, P. K. Jain, I. H. El-Sayed, and M. A. El-Sayed, Nanomedicine 2, 681 (2007).

${ }^{10}$ M. S. Yavuz, Y. Y. Cheng, J. Y. Chen, C. M. Cobley, Q. Zhang, M. Rycenga, J. W. Xie, C. Kim, K. H. Song, A. G. Schwartz, L. H. V. Wang, and Y. N. Xia, Nature Mater. 8, 935 (2009).

${ }^{11}$ M. Thomas and A. M. Klibanov, Proc. Natl. Acad. Sci. U. S. A. 100, 9138 (2003).

${ }^{12}$ J. R. Heath and M. E. Davis, Annu. Rev. Med. 59, 251 (2008).

${ }^{13}$ N. Nishiyama, Nat. Nanotechnol. 2, 203 (2007).

${ }^{14}$ T. Taniyama, E. Ohta, and T. Sato, Europhys. Lett. 38, 195 (1997).

${ }^{15}$ M. J. Zhu, D. M. Bylander, and L. Kleinman, Phys. Rev. B 42, 2874 (1990).

${ }^{16}$ S. Blügel, Phys. Rev. Lett. 68, 851 (1992).

${ }^{17}$ S. Blügel, Phys. Rev. B 51, 2025 (1995).

${ }^{18}$ B. Sampedro, P. Crespo, A. Hernando, R. Litrán, J. C. Sánchez López, C. López Cartes, A. Fernandez, J. Ramírez, J. González Calbet, and M. Vallet, Phys. Rev. Lett. 91, 237203 (2003).

${ }^{19}$ T. Shinohara, T. Sato, and T. Taniyama, Phys. Rev. Lett. 91, 197201 (2003). 
${ }^{20}$ Y. Yamamoto, T. Miura, M. Suzuki, N. Kawamura, H. Miyagawa, T. Nakamura, K. Kobayashi, T. Teranishi, and H. Hori, Phys. Rev. Lett. 93, 116801 (2004).

${ }^{21}$ H. Hori, Y. Yamamoto, T. Iwamoto, T. Miura, T. Teranishi, and M. Miyake, Phys. Rev. B 69, 174411 (2004).

${ }^{22}$ X. Liu, M. Bauer, H. Bertagnolli, E. Roduner, J. van Slageren, and F. Phillipp, Phys. Rev. Lett. 97, 253401 (2006).

${ }^{23}$ B. D. Cullity and C. D. Graham, in Introduction to Magnetic Materials, 2nd ed. (IEEE Press, Piscataway, NJ, 2009).

${ }^{24}$ K. Kuribayashi and S. Kitamura, Thin Solid Films 400, 160 (2001).

${ }^{25}$ L. Wang and Y. Yamauchi, Chem. - Eur. J. 17, 8810 (2011).
${ }^{26}$ L. Wang and Y. Yamauchi, J. Am. Chem. Soc. 131, 9152 (2009).

${ }^{27}$ G. M. Bancroft, I. Adams, L. L. Coatsworth, C. D. Bennewitz, J. D. Brown, and W. D. Westwood, Anal. Chem. 47, 586 (1975).

${ }^{28}$ T. L. Barr, J. Phys. Chem. 82, 1801 (1978).

${ }^{29}$ P. Crespo, R. Litrán, T. C. Rojas, M. Multigner, J. M. de la Fuente, J. C. Sánchez-López, M. A. García, A. Hernando, S. Penadés, and A. Fernández, Phys. Rev. Lett. 93, 087204 (2004).

${ }^{30}$ M. Pereiro, D. Baldomir, and J. E. Arias, Phys. Rev. A 75, 063204 (2007).

${ }^{31}$ Y. Yang, O. Sugino, and T. Ohno, Phys. Rev. B 85, 035204 (2012).

${ }^{32}$ H. T. Zhang, J. Ding, and G. M. Chow, Langmuir 24, 375 (2008). 